\title{
Central role of RAGE-dependent neointimal expansion in arterial restenosis
}

\author{
Taichi Sakaguchi, ${ }_{1}^{1}$ Shi Fang Yan, ${ }^{1}$ Shi Du Yan, ${ }^{2}$ Dmitri Belov, ${ }^{1}$ Ling Ling Rong, \\ Monica Sousa, ${ }^{1}$ Martin Andrassy, ${ }^{1}$ Steven P. Marso, ${ }^{3}$ Stephan Duda, ${ }^{4}$ Bernd Arnold, ${ }^{4}$ \\ Birgit Liliensiek, ${ }^{4}$ Peter P. Nawroth, ${ }^{4}$ David M. Stern, ${ }^{1,5}$ Ann Marie Schmidt, ${ }^{1}$ \\ and Yoshifumi Naka ${ }^{1}$
}

\author{
${ }^{1}$ Department of Surgery and \\ ${ }^{2}$ Department of Pathology, College of Physicians \& Surgeons, Columbia University, New York, New York, USA \\ ${ }^{3}$ Mid-America Heart Institute, Kansas City, Missouri, USA \\ ${ }^{4}$ Department of Medicine and Cancer Research Institute, University of Heidelberg, Heidelberg, Germany \\ ${ }_{5}^{5}$ Department of Physiology \& Cellular Biophysics, College of Physicians \& Surgeons, Columbia University, New York, \\ New York, USA
}

Cellular proliferation, migration, and expression of extracellular matrix proteins and MMPs contribute to neointimal formation upon vascular injury. Wild-type mice undergoing arterial endothelial denudation displayed striking upregulation of receptor for advanced glycation end products (RAGE) in the injured vessel, particularly in activated smooth muscle cells of the expanding neointima. In parallel, two of RAGE's signal transducing ligands, advanced glycation end products (AGEs) and S100/calgranulins, demonstrated increased deposition/expression in the injured vessel wall. Blockade of RAGE, employing soluble truncated receptor or antibodies, or in homozygous RAGE null mice, resulted in significantly decreased neointimal expansion after arterial injury and decreased smooth muscle cell proliferation, migration, and expression of extracellular matrix proteins. A critical role for smooth muscle cell RAGE signaling was demonstrated in mice bearing a transgene encoding a RAGE cytosolic tail-deletion mutant, specifically in smooth muscle cells, driven by the SM22 $\alpha$ promoter. Upon arterial injury, neointimal expansion was strikingly suppressed compared with that observed in wild-type littermates. Taken together, these data highlight key roles for RAGE in modulating smooth muscle cell properties after injury and suggest that RAGE is a logical target for suppression of untoward neointimal expansion consequent to arterial injury.

J. Clin. Invest. 111:959-972 (2003). doi:10.1172/JCI200317115.

\section{Introduction}

Expansion of the neointima is a unifying facet of innate chronic atherosclerosis and the response to acute arterial injury, such as that induced by angioplasty. Restenosis after vascular injury limits the long-term benefit of this intervention in many human subjects, even in the setting of interventions such as stenting and administration of anti-platelet or anti-inflammatory agents

Received for publication October 11, 2002, and accepted in revised form January 27, 2003.

Address correspondence to: Yoshifumi Naka, New York Presbyterian Hospital, Milstein Hospital Building, Suite 7-435, 177 Fort Washington Avenue, New York, New York 10032, USA. Phone: (212) 305-0828; Fax: (212) 305-2439;

E-mail:yn33@columbia.edu.

Conflict of interest: Ann Marie Schmidt is a consultant for and receives research support from TransTech Pharma.

Nonstandard abbreviations used: smooth muscle cell (SMC); mononuclear phagocyte (MP); receptor for advanced glycation end products (RAGE); advanced glycation end product (AGE); soluble RAGE (sRAGE); Janus kinase-signal transducers and activators of transcription (JAK/STAT); transgenic (Tg); dominant negative (DN); internal elastic lamina (IEL); external elastic lamina (EEL); carboxy(methyl) lysine (CML); myeloperoxidase (MPO); murine serum albumin (MSA); intimal/ medial (I/M); extracellular signal-regulated protein kinase (Erk); phosphoinositide 3-kinase (PI3K); protein kinase B (PKB).
(1-4). Recently, the finding that sirolimus displayed potent antimitotic effects in vascular smooth muscle cells (SMCs) led to the testing of this agent in vivo; in experimental models, sirolimus-coated stents led to significantly reduced neointimal expansion in pig/rabbit models $(5,6)$. In human subjects, at 6 months after angioplasty, strikingly reduced neointimal expansion and restenosis was observed versus therapy using standard stents (7). Although systemic toxicity of this agent, however, precludes it for extended use in innate, longstanding disease of the systemic vasculature, these observations highlighted key roles for SMCs in the pathologic expansion of the neointima that ultimately impinges on the vascular lumen (8-14). Taken together with findings suggesting important roles for mononuclear phagocytes (MPs) in magnifying cellular activation and inflammation within the injured vessel wall (15, 16), these considerations led us to hypothesize an important role for receptor for advanced glycation end products (RAGE) in arterial injury.

RAGE is a multiligand member of the immunoglobulin superfamily expressed at low levels in adult tissues in homeostasis, but highly upregulated at sites of vascular pathology (17-19). Ligand-triggered RAGEdependent cellular activation augments inflammatory 
responses and enhances cellular migration and proliferation (20-25). In atherosclerosis, pharmacologic blockade of the receptor stabilized established atherosclerosis in both euglycemic and diabetic mice deficient in apoE in the absence of alterations in glucose levels or lipid number/profile $(17,18)$. These considerations led us to test the hypothesis that activation of RAGE in the acutely injured arterial wall might contribute to pathologic neointimal expansion.

Here, employing a murine model of arterial endothelial denudation, we show that RAGE and two of its ligands, advanced glycation end products (AGEs) and S100/calgranulins, are upregulated at the site of vascular injury, particularly within the expanding neointima. Inhibition of RAGE using a soluble, truncated form of the receptor (sRAGE) and anti-RAGE Ab's, as well as genetically manipulated mice, strongly suppressed neointimal formation in mice upon arterial denudation. In parallel, pathways critically modulated by RAGE signaling were central for intimal SMC proliferation, migration, and expression of ECM molecules. These events involved, at least in part, the Janus kinase-signal transducers and activators of transcription (JAK/STAT) pathway. These findings highlight the concept that RAGE importantly contributes to the biologic properties of proliferative/migratory SMCs and suggest that the beneficial effects of blockade of this axis might extend beyond chronic systemic atherosclerosis, but, as well, to the distinct lesion induced at the site of acute vascular injury.

\section{Methods}

Animals and induction of vascular injury. Male C57BL/6 mice were purchased from The Jackson Laboratory (Bar Harbor, Maine, USA). Transgenic (Tg) mice were generated to drive expression of a signaling deficient mutant of RAGE (dominant negative, or DN), DN RAGE, in SMCs using an 445-bp prion of the SM22 $\alpha$ promoter (26). $\mathrm{Tg}$ cassettes were created by releasing a vector backbone using unique restriction enzymes (NotI and PmeI), and these were injected directly into mouse C57BL/6 oocytes. After mating with males, founders were identified by Southern blot analysis, and transmission of the transgene was verified. In our experiments, hemizygous Tg mice and wild-type littermates (all in C57BL/6 background) were employed. Homozygous RAGE null mice (generated in the 129 strain) were backcrossed three generations into C57BL/6; mating of heterozygous RAGE null males and females yielded heterozygous mice, as well as wildtype RAGE-bearing $\left(\mathrm{RAGE}^{+/+}\right)$and homozygous RAGE null animals (RAGE-/-). In all cases, littermates (wild type) were employed as controls.

The apoE-deficient mice (27) were purchased from The Jackson Laboratory (previously backcrossed more than ten generations into $\mathrm{C} 57 \mathrm{BL} / 6$ ).

All procedures were approved by the Institutional Animal Care and Use Committee at Columbia University. Mice (male: age 8-12 weeks) were anesthetized with intraperitoneal injections of ketamine $(50 \mathrm{mg} / \mathrm{kg})$ and xylazine $(5 \mathrm{mg} / \mathrm{kg})$. A groin incision was made, and the common femoral artery was clamped at the level of the inguinal ligament; an arteriotomy was made distal to the epigastric branch. An angioplasty guide wire (0.25-mm diameter; Advanced Cardiovascular Systems Inc., Santa Clara, California, USA) (28) was inserted and placed in the femoral artery distal to the inferior epigastric artery. The clamp was removed, and the endoluminal denudation was induced by three passages of the guide wire (28). In this model, denudation was limited to the intima. In instrumented arteries, vessels that had been subjected to excessive injury (beyond the intima) were readily apparent because the internal elastic lamina (IEL) was not visible. There arteries were immediately excluded from consideration. After removal of the wire, the arteriotomy site was ligated.

Mice were treated with sRAGE, mouse serum albumin (Sigma-Aldrich, St. Louis, Missouri, USA), antiRAGE $\mathrm{F}\left(\mathrm{ab}^{\prime}\right)_{2}$, or nonimmune $\mathrm{F}\left(\mathrm{ab}^{\prime}\right)_{2}$ at the indicated concentrations once daily by an intraperitoneal route. For BrdU labeling, mice received two intraperitoneal injections of BrdU (2.5 mg/injection; Sigma-Aldrich) $12 \mathrm{~h}$ and $1 \mathrm{~h}$ before sacrifice.

RAGE-related reagents. Murine soluble RAGE and rabbit anti-murine RAGE IgG were prepared and characterized as described $(17,22)$. $\mathrm{F}\left(\mathrm{ab}^{\prime}\right)_{2}$ fragments were prepared from rabbit IgG using a kit from Pierce Chemical Co. (Rockford, Illinois, USA). All materials were devoid of contaminating endotoxin Detoxi-gel columns (Pierce Chemical Co.) and then were reassayed for endotoxin contamination using the limulus amebocyte assay (Sigma-Aldrich).

Tissue analyses. Harvesting of vessel segments was performed by perfusion fixation for $5 \mathrm{~min}$ at physiologic pressure with formalin (10\%). Specimens were fixed for $24 \mathrm{~h}$ in formalin (10\%), segments were cut transversely at 3-mm intervals from the inguinal ligament to the epigastric artery, and tissues were embedded in paraffin. The section $(5 \mu \mathrm{m})$ at the midportion of each femoral artery was treated with Van Gieson's elastic staining kit (Sigma-Aldrich), and the degree of intimal thickening was analyzed quantitatively using a Zeiss microscope and image analysis system (Media Cybernetics Inc., Silver Spring, Maryland, USA). Three types of measurements were made, all performed by one of the investigators blinded to the experimental protocol, including assessment of luminal area, the area encircled by the IEL, and the area encircled by the external elastic lamina (EEL). Intimal area was calculated by subtracting the area encircled by IEL from that encircled by the EEL. Image analysis of Masson's trichrome sections was performed by determining the area occupied by bluestained matrix using a chromogen-separating technique and analytical software (Adobe Photoshop 5.0; Adobe Systems Inc., Mountain View, California, USA).

Representative sections were stained with monoclonal anti-smooth muscle $\alpha$-actin IgG (1:100; SigmaAldrich), rat monoclonal anti-mouse F4/80 IgG (1:50; 
Caltag Laboratories Inc., Burlingame, California, USA), rabbit anti-human S100b IgG (1:25; DakoCytomation, Glostrup, Denmark), and affinity-purified guinea pig anti-AGE IgG prepared against AGE-modified keyhole limpet hemocyanin $(5 \mu \mathrm{g})(20,29)$. Sections were deparaffinized and blocked with hydrogen peroxide $(3 \%)$ in methanol for $10 \mathrm{~min}$. Blocking was performed with goat serum (4\%) and BSA (1\%) in PBS. Primary $A b$ 's were added to slides and incubated overnight at $4^{\circ} \mathrm{C}$. Secondary Ab's (1:100 affinity-purified biotinylated anti-mouse, rat, guinea pig, or rabbit IgG; SigmaAldrich) were added for $30 \mathrm{~min}$. at room temperature. Sections were reacted with HRP-conjugated streptavidin (1:100; Sigma-Aldrich) for $30 \mathrm{~min}$. at room temperature and developed with either 3,3'-diaminobenzidine (DAB substrate kit; Vector Laboratories, Burlingame, California, USA) or 3-amino-9-ethylcarbazole (AEC substrate kit; Vector Laboratories). Counterstaining was performed with Mayer's hematoxylin solution (Sigma-Aldrich). Masson's trichrome stain was performed according to standard techniques and quantified by image analysis, as discussed above.

SMC replication in the media and intima of arterial segments was evaluated by staining sections with rat monoclonal anti-BrdU IgG (1:100; Accurate Chemical $\&$ Scientific Co., Westbury, New York, USA). Numbers of stained nuclei and total nuclei in the neointima were counted, and a BrdU-labeling index was calculated (BrdU-labeled nuclei/total nuclei $\times 100 \%$ ). Apoptotic cells were visualized by the TUNEL method (Apotag kit; Intergen Co., Purchase, New York, USA) applied to adjacent sections from the above experiment for assessment of BrdU positivity. TUNEL-positive cells were quantified, and an index of TUNEL-positive cells was determined by the same method described for the BrdU-labeling index above.

Immunoblotting and immunoprecipitation. At least eight arteries were snap-frozen in liquid nitrogen, pooled, and stored at $-80^{\circ} \mathrm{C}$. Tissue samples were ground to a fine powder under liquid nitrogen and incubated in ice-cold lysis buffer (Cell Signalling Technology, Beverly, Massachusetts, USA). Protein extracts of cultured cells were prepared with the same lysis buffer. Particulate material was removed by centrifugation, and protein concentration was determined by the Bio-Rad protein assay (Bio-Rad Laboratories, Hercules, California, USA). Equal amounts of total protein $(10-30 \mu \mathrm{g} / \mathrm{sam}$ ple) were subjected to SDS-PAGE (7.5-12\%) followed by electrophoretic transfer to nitrocellulose membranes (30). Nonspecific binding was blocked by incubation of membranes with nonfat dry milk (5\%) for $1 \mathrm{~h}$. Blots were incubated with rabbit anti-human RAGE IgG (22), rabbit anti-Erk1/2 (Promega Corp., Madison, Wisconsin, USA), anti-phospho-Erk1/2 IgG (Cell Signalling Technology), anti-Akt IgG (Cell Signaling Technology), anti-phospho-Akt IgG (Cell Signalling Technology), anti-Jak2 IgG (Santa Cruz Biotechnology Inc., Santa Cruz, California, USA), anti-phospho Jak2 IgG (Affinity BioReagents Inc., Golden, Colorado, USA), anti-
Stat3 IgG (Santa Cruz Biotechnology Inc.), or anti-phospho-Stat3 IgG (Cell Signalling Technology), each at a dilution of 1:1,000 for $3 \mathrm{~h}$. HRP-conjugated donkey anti-rabbit IgG secondary Ab (1:2,000, Santa Cruz Biotechnology Inc.) was used to identify sites of binding of the primary $\mathrm{Ab}$.

Complexes of sRAGE- and AGE-immunoreactive material were identified in the plasma of animals treated with sRAGE by immunoprecipitation with antiRAGE IgG followed by immunoblotting with affinitypurified anti-AGE IgG $(20,29)$. In brief, serum $(25 \mu \mathrm{l})$ from mice subjected to arterial injury, treated with either vehicle, or sRAGE, or sham controls, was diluted 1:1 with PBS and incubated overnight at $4^{\circ} \mathrm{C}$ with antiAGE IgG (10-20 $\mu \mathrm{g} / \mathrm{ml})$. Protein A/G (7 $\mu \mathrm{l}$; Pierce Chemical Co.) was added for $1 \mathrm{~h}$ at room temperature, followed by three washes in Tris-buffered saline with Tween 20 (0.05\%). After centrifugation, the pellet was solubilized in reduced SDS sample buffer $(40 \mu \mathrm{l})$ and subjected to SDS-PAGE (10\%) under reducing conditions. Proteins were electrophoretically transferred to nitrocellulose, membranes were blocked with nonfat dry milk (10\%), and then incubated with primary $\mathrm{Ab}$ for $3 \mathrm{~h}$ at $37^{\circ} \mathrm{C}$ (rabbit anti-RAGE IgG; $4 \mu \mathrm{g} / \mathrm{ml}$ ) followed by secondary $\mathrm{Ab}$ (peroxidase-conjugated donkey anti-rabbit IgG, 1:40,000; The Jackson Laboratory) for $1 \mathrm{~h}$ at room temperature.

$R N A$ extraction and RT-PCR. Total RNA was extracted from at least eight vessels prepared as for immunoblotting above, or from lung tissue in the case of RAGE null and control mice, except that Trizol reagent (Life Technologies Inc., Rockville, Maryland, USA) was employed. Reverse transcription was done with Superscript II (Life Technologies Inc.), primed by oligo-dT, following the protocol suggested by the manufacturer. The following primers were used: for mouse RAGE, sense ( $5^{\prime}$-AGCGGCTGGAATGGAAACTGAACA- $3^{\prime}$ ) and antisense (5'-GAAGGGGCAAGGGCACACCATC- $3^{\prime}$ ); for mouse matrix metalloproteinase 12 , sense ( $5^{\prime}$-GCAGTGCCCCAGAGGTCAAG- $3^{\prime}$ ) and antisense ( $5^{\prime}$-TGCTGGGGTTAAGGTATCTGTAGG-3'); for mouse S100, sense (5'-GCTGACCACCATGCCCCTGTAG$\left.3^{\prime}\right)$ and antisense (5'-CTGGCCATTCCCCTCCTCTGTC$\left.3^{\prime}\right)$; and for mouse tenascin-C, sense (5'-CGGGATCTTCGACATGTTTACTAT- $3^{\prime}$ ) and antisense (5'-CCTTCCGCGGCTTATTCC- $\left.3^{\prime}\right)$. To control for differences in total RNA, $\beta$-actin transcripts were visualized by RT-PCR using as primers sense ( $5^{\prime}$-GTGGGCCGCTCTAGGCACCAA- $\left.3^{\prime}\right)$ and antisense (5'-CTCTTTGATGTCACGCACGATTTC- $\left.3^{\prime}\right)$. For studies to analyze expression of transcripts from $\mathrm{Tg}$ DN-RAGE mice, the following primers were used for human RAGE: (a) spanning a portion of the extracellular domain, sense (5'-GGAAGCCCCTGGTGCCTAATGA-3') (482-503 bp) and antisense ( $5^{\prime}$-GCCCCTCCTCGCCTGGTTC-3') (964-982 bp); and (b) spanning from the transmembrane domain to the cytosolic tail, sense $\left(5^{\prime}\right.$-AGGATCAGGGCTGGGAACTCTA-3') (1002-1023 bp) and antisense (5'-TCCCCCTGAACCTGAAACATAAAA-3') (poly A tail). 
Assessment of myeloperoxidase activity. Femoral arteries were retrieved rapidly from mice at sacrifice and homogenized in hexadecyltrimethylammonium bromide followed by freeze/thaw cycles to release myeloperoxidase from leukocyte granules. Myeloperoxidase activity was measured as described previously (31).

Cell culture and in vitro assays on cultured SMCs. Mouse vascular SMCs were cultured from the aortas using a modification of the procedure of Tarvo and Barret (32). Experiments were conducted on SMCs after five to seven passages in culture. Cultures were composed of more than $95 \%$ SM- $\alpha$-actin based on immunostaining.

Migration assays were performed using 48-well microchemotaxis chambers (Neuro-Probe Inc., Gaithersburg, Maryland, USA) containing a polycarbonate membrane with $8-\mu \mathrm{m}$ pores (Neuro-Probe Inc.). For cell migration studies, suspensions of SMCs $\left(2 \times 10^{4}\right.$ cells/well) were prepared and added to the upper compartment of the microchemotaxis chamber, and chemotactic agents were placed in the lower and/or upper compartments (S100b; Calbiochem-Novabiochem Corp., San Diego, California, USA). Chambers were incubated for 4 hours at $37^{\circ} \mathrm{C}$ in a humidified $5 \%$ carbon dioxide/air atmosphere. Cells attached to the upper side of the filter were removed manually by scraping, and cells migrating through pores of the membrane and emerging on the lower side were visualized by staining with Coomassie blue (Sigma-Aldrich). The latter cells were counted under the light microscope $(\times 400)$. For each filter, the number of cells in three adjacent fields was counted, and the average was determined. Migrating SMCs were quantified from at least four separate filters, and the results were averaged. The latter average was considered to represent the number of cells migrating across the filter under a particular condition. The migration assay was repeated twice with different SMC preparations from the pooled cell lines with consistent results. In experiments using anti-RAGE IgG, nonimmune IgG, and AG490 (Calbiochem-Novabiochem Corp.), cells were preincubated with these agents or vehicle alone for $2 \mathrm{~h}$ (Ab's) or $30 \mathrm{~min}$ (AG490) at $37^{\circ} \mathrm{C}$ prior to the addition to microchemotaxis chambers.

Proliferation of cultured SMCs was quantified by ${ }^{3} \mathrm{H}$-thymidine incorporation. SMCs were seeded at a density of $2 \times 10^{4}$ cells/well in 24-well tissue culture-treated plates and incubated in serum-free DMEM for $48 \mathrm{~h}$. Following a 120-minute preincubation with the indicated concentration of anti-RAGE IgG, nonimmune IgG, or AG490, cells were exposed to serum-free DMEM containing the indicated concentration of S100b along with ${ }^{3} \mathrm{H}$-thymidine $(1 \mu \mathrm{Ci} /$ well $)$. After the incubation period, cells were harvested 48 hours later, and cellular proliferation was determined based on the incorporation of ${ }^{3} \mathrm{H}$-thymidine. Cell counting confirmed that increased ${ }^{3} \mathrm{H}$-thymidine incorporation reflected an increase in cell number as described previously (33). For positive control to test the ability of SMCs to migrate/proliferate, 100 $\mathrm{ng} / \mathrm{ml}$ platelet-derived growth factor (PDGF-BB; SigmaAldrich) was employed.
Expression of tenascin-C transcripts was assessed upon exposure of cultured murine SMCs $\left(2 \times 10^{5}\right.$ cells $)$ for $12 \mathrm{~h}$ at $37^{\circ} \mathrm{C}$ to $\mathrm{S} 100 \mathrm{~b}(5 \mu \mathrm{g} / \mathrm{ml})$ alone or following preincubation for $2 \mathrm{~h}$ with anti-RAGE IgG $(10 \mu \mathrm{g} / \mathrm{ml})$ or nonimmune IgG $(10 \mu \mathrm{g} / \mathrm{ml})$. Total RNA was isolated and subjected to RT-PCR as above.

Statistical analysis. All data are expressed as the mean plus or minus the SE of the mean. All analyses were performed using the Statview Statistical package (version j4.5). To determine statistical significance, the Student unpaired $t$ test was used for a comparison between groups. ANOVA with post-hoc analysis with the Bonferroni/Dunn test was used to compare three or more groups. $P$ values of less than 0.05 were considered statistically significant.

\section{Results}

RAGE and its ligands, AGEs and S100/calgranulins, are upregulated in the vessel wall upon acute arterial injury. Femoral artery denudation injury in C57BL/6 mice was accomplished upon three passages of an angioplasty guide wire. To examine expression of RAGE and its ligands after injury, we first performed RT-PCR. Compared with sham (uninstrumented control) vessels, RAGE transcripts were increased by day 3 after injury and remained elevated in the vessel through day 28 (Figure 1a). Immunostaining of the injured arterial segment demonstrated upregulation of RAGE within the neointimal and medial cells by day 4 in a distribution overlapping that of the SMC marker, $\alpha$-actin (Figure $1 \mathrm{~b}$; right-most panel is staining for $\alpha$-actin). Consistent with previous observations, very few MPs were noted in the neointima or media after arterial injury in wild-type C57BL/ 6 mice at 4, 7, or 14 days after injury, as assessed by staining with Ab's to the MP marker, F4/80 (data not shown) (28).

To determine if enhanced ligand formation/deposition in the injured arterial segment might contribute to RAGE-dependent cellular activation, we assessed expression of ligands for the receptor in the vessel wall. RT-PCR showed induction of S100 transcripts by 3 days after injury, which, as in the case of RAGE, persisted through 28 days after acute injury (Figure 1a). In the vessel wall, immunohistochemistry performed with polyclonal $\mathrm{Ab}$ reactive with $\mathrm{S} 100 \mathrm{~b}$ demonstrated S100 antigen throughout the intima and media of the damaged vessel (Figure 1c). These findings are consistent with multiple previous studies demonstrating that S100s are upregulated by injury, such as that induced by cutaneous wounding, or by acute cellular activation induced by rapidly acting mediators such as LPS (34-37).

In addition to S100/calgranulins, we sought evidence for generation of AGEs, another class of RAGE ligand (25) within the injured vessel wall. We employed an affinity-purified $\mathrm{Ab}$ to an heterogeneous group of AGEs, the principal immunoreactive epitope of which was carboxy(methyl) lysine-modified (CML-modified) adducts (25). Although at baseline, few if any immunoreactive AGE epitopes were evident in the ves- 
sel wall, immunohistochemistry revealed an increase in AGE deposition/formation upon injury. AGEs were observed in the neointima within 4 days of arterial injury and persisted until day 21 (Figure 1d), consistent with previous findings in a model of balloon injury-induced damage to the rat carotid artery (38). Furthermore, to identify potential mechanisms by which AGEs might be generated upon acute arterial injury in euglycemic mice, we sought evidence for generation of oxidant stress in the injured vessel wall, a known trigger of AGE formation, even in the absence of elevated levels of glucose. Compared with sham treatment, arterial injury resulted in a significant increase in myeloperoxidase (MPO) activity at $1 \mathrm{~h}$ after guide-wire injury (Figure 1e). These findings bear significance in at least two settings; first, enhanced MPO activity is linked to generation of oxidant stress, and second, increased MPO activity is linked directly to generation of CML-AGEs (39-40). Taken together, these observations provide a mechanism by which acute arterial injury induces generation of AGEs; together with increased expression of S100/calgranulins, we hypothesized that the overlapping expression of these ligands with RAGE sets the stage for RAGE-driven enhanced cellular activation and neointimal expansion after injury.

\section{Figure 1}

Expression of RAGE and S100/calgranulins and accumulation of AGEs following arterial injury. (a) Femoral artery guide-wire injury was performed in C57BL/ 6 wild-type mice, and RNA was harvested for RT-PCR using primers for RAGE, $\mathrm{S} 100$, and $\beta$-actin at the indicated times. (b-d) Immunohistochemistry was performed on femoral artery segments harvested at the injury site using an $A b$ to RAGE (b), S100b (c), or AGE (d). In b, sections were stained with $A b$ to smooth muscle $\alpha$-actin. Scale bar: $50 \mu \mathrm{m}$. (e) Myeloperoxidase activity. Wild-type mice were subjected to arterial injury; $1 \mathrm{~h}$ after injury, vessel segments from the injured versus uninstrumented controls were retrieved and assayed for MPO activity. For each point, vessels were harvested and pooled from $n=2$ mice per condition. Finally, data from three sets of pooled animals per condition are reported.

e
Blockade of ligand-RAGE interaction suppresses neointimal expansion. Based on the expression/upregulation of RAGE and its ligands after injury, we tested whether blockade of ligand-RAGE interaction might impact on neointimal expansion. First, we blocked ligand-RAGE binding by administration of sRAGE, the truncated extracellular ligand-binding domain of the receptor in C57BL/6 mice. Soluble RAGE was administered from the day prior to injury (day 0 ) through postinjury day 7 , and animals were studied up to day 28 . Compared with mice treated with vehicle, murine serum albumin (MSA), mice receiving sRAGE, $100 \mu \mathrm{g}$, displayed significantly decreased neointimal expansion, as assessed by intimal/medial (I/M) ratio over 1, 2, 3, and 4 weeks after injury (Figure $2 a$; representative sections illustrated by van Gieson's elastic stain from MSA- and sRAGE-treated mice are shown in Figure 2, $\mathrm{b}$ and $\mathrm{c}$, respectively). The effects of sRAGE were dose a

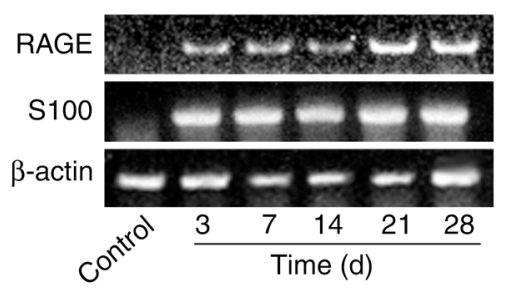

b

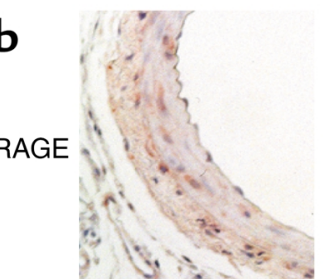

c

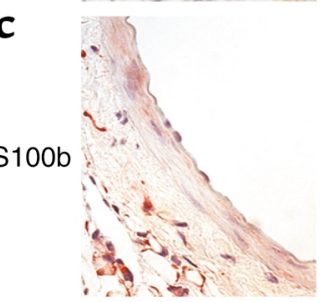

d
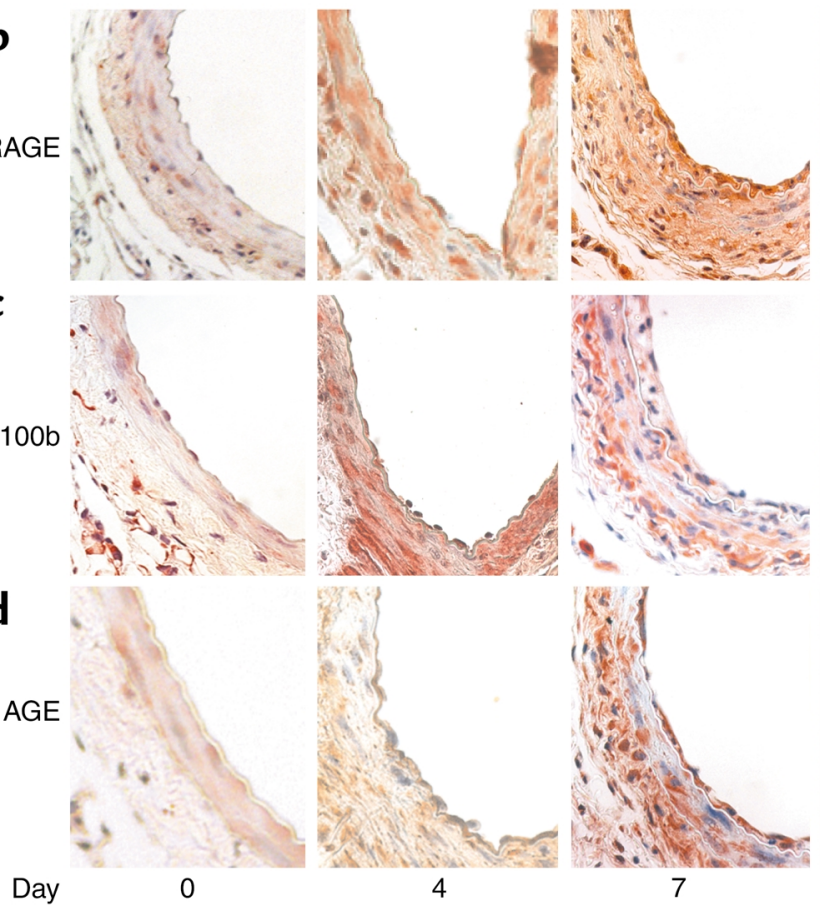

Day

4

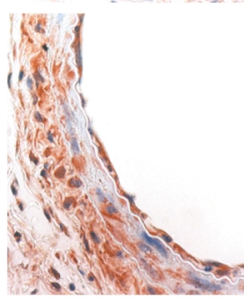

7
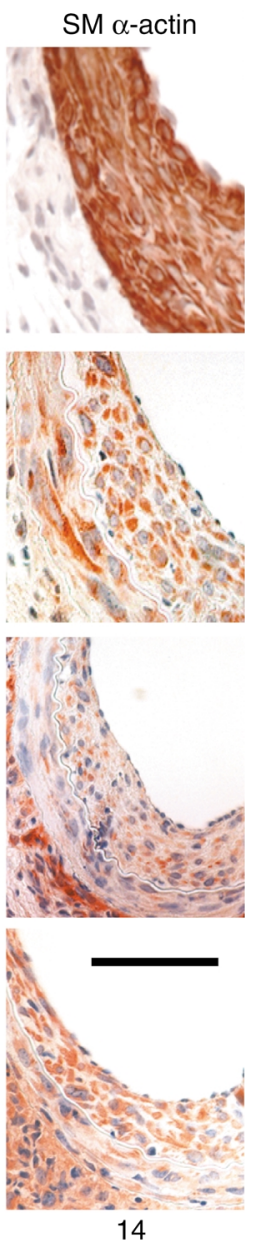

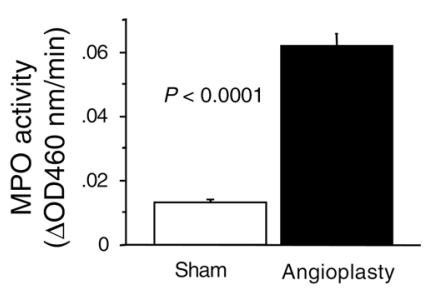



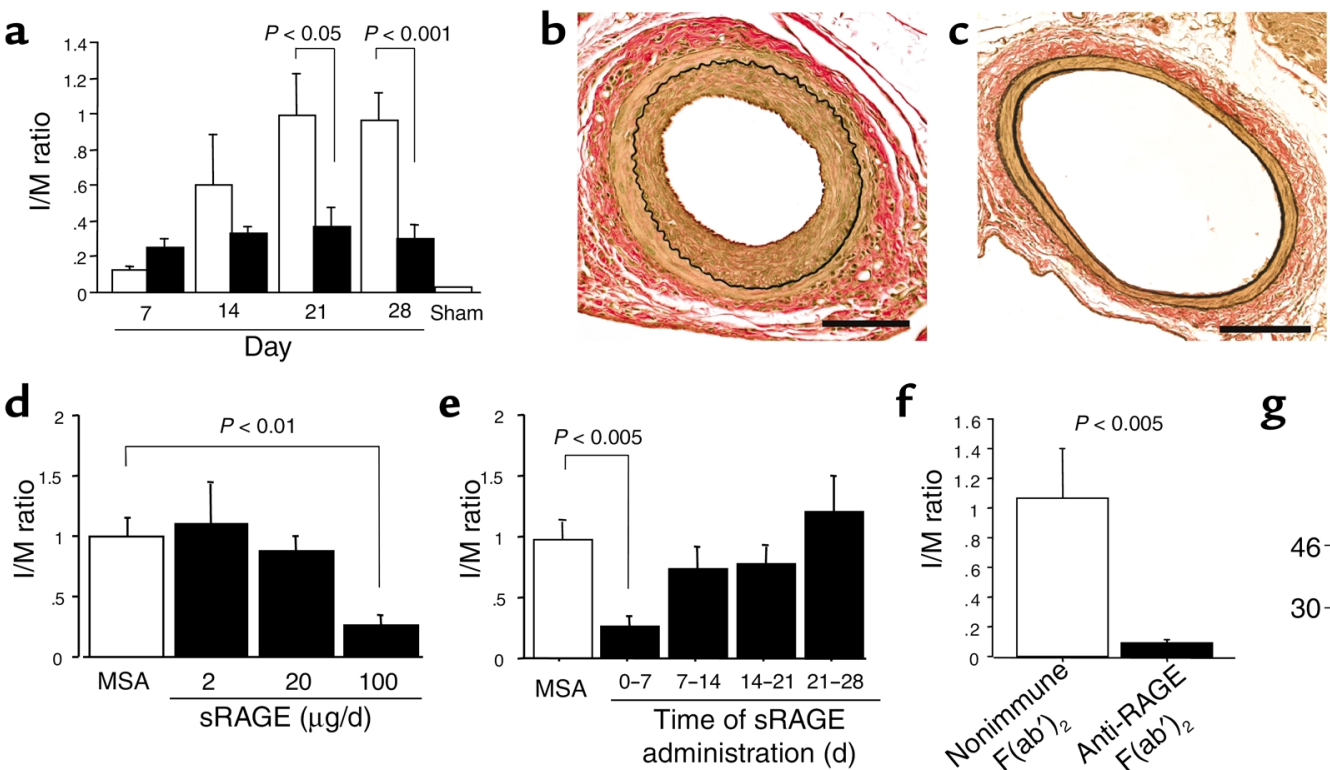

g

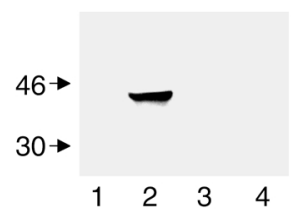

\begin{abstract}
Figure 2
RAGE blockade and neointimal expansion after acute arterial injury. (a-c) Femoral artery guide-wire injury was performed, and animals were followed until the indicated day, during which time they were treated with either sRAGE (100 $\mu \mathrm{g} / \mathrm{day}$, intraperatoneally; black bars) or MSA $(100 \mu \mathrm{g} /$ day; white bars) from days 0 to 7 (day 1 is the day of injury). I/M was determined (a) or vessels were stained with van Gieson's elastic stain. (b) MSA. (c) sRAGE. Scale bar: $50 \mu \mathrm{m}$. (d) Animals were treated with the indicated dose of sRAGE or MSA followed by harvest of the vessels on day 28 , and $\mathrm{I} / \mathrm{M}$ ratio was determined. (e) Animals were subjected to femoral artery injury and then treated with sRAGE over the indicated interval. At 28 days, animals were sacrificed and I/M ratio determined. (f) Animals were subjected to femoral artery injury and received $\mathrm{F}\left(\mathrm{ab}^{\prime}\right)_{2}(100 \mu \mathrm{g} /$ day, intraperitoneally; days 0-7) prepared from either nonimmune rabbit IgG or rabbit anti-RAGE IgG. (g) Serum from sRAGEtreated mice subjected to femoral artery injury was immunoprecipitated with anti-AGE IgG; immune precipitate was solubilized and subjected to Western blotting with anti-RAGE IgG. Lane 1, MSA treatment from days 0 to 3; lane 2, sRAGE from days 0 to 3; lane 3, normal mouse serum without injury; lane 4, sRAGE treatment and immunoprecipitation with nonimmune IgG. In (a-f) at least 10 to 20 vessels per condition were analyzed by an investigator blinded to the experimental conditions. The immunoprecipitation study ( $g$ ) was performed three times.
\end{abstract}

dependent; although $100 \mu \mathrm{g} /$ day from day 0 through postinjury day 7 exerted a significant impact on suppression of neointimal expansion, animals receiving either 2 or $20 \mu \mathrm{g}$ sRAGE per day failed to display statistically significant decreases in $\mathrm{I} / \mathrm{M}$ ratio (Figure 2d). Furthermore, RAGE-dependent mechanisms impacting on neointimal expansion were most relevant in the days immediately following injury; when sRAGE administration was begun 7 days or more after arterial injury, no beneficial impact on neointimal expansion was observed (Figure 2e). Soluble RAGE exerts its effects indirectly, by blocking ligand engagement of the cell surface receptor. To directly block the receptor and assess the impact on neointimal expansion, $\mathrm{F}\left(\mathrm{ab}^{\prime}\right)_{2}$ fragments of anti-RAGE IgG (and nonimmune rabbit $\operatorname{IgG})$ were prepared $(21,22)$. Administration of antiRAGE $\mathrm{F}\left(\mathrm{ab}^{\prime}\right)_{2}$ from days 0-7 (injury, day 1) resulted in significant suppression of $\mathrm{I} / \mathrm{M}$ ratio compared with animals treated on the same schedule with nonimmune rabbit $\mathrm{F}\left(\mathrm{ab}^{\prime}\right)_{2}$ (Figure 2f). These data implicate ligand-RAGE interaction in the vascular response to arterial injury. Direct support for the interaction of RAGE with its ligands was obtained by immunoprecipitating serum from sRAGE-treated animals with affinity-purified anti-AGE IgG, followed by immunoblotting with $\mathrm{Ab}$ immunoreactive with RAGE. By
Western blot analysis, an immunoreactive RAGE band with molecular mass of approximately $40 \mathrm{kDa}$ was observed on day 3 after injury in sRAGE-treated mice with affinity-purified anti-AGE IgG followed by immunoblotting with anti-RAGE IgG (Figure 2g, lane 2), but not in mice treated with MSA (Figure $2 \mathrm{~g}$, lane 1). In Figure 2g, lane 3 shows normal mouse serum subjected to the same procedure of immunoprecipitation/blotting procedure as control. Importantly, when anti-AGE IgG was replaced with nonimmune IgG in the immunoprecipitation step of serum from sRAGEtreated mice 3 days after arterial injury, no band immunoreactive with RAGE was observed on Western blotting with anti-RAGE IgG (Figure 2g, lane 4).

Homozygous RAGE null mice and mice expressing signaling deficient mutation DNRAGE in SMCs display decreased neointimal expansion upon arterial injury. In addition to pharmacologic blockade of the ligand-receptor interaction, our next studies were focused on employing genetically modified mice to test the role of RAGE and its signaling in SMCs in the response to arterial injury. In the former case, we employed homozygous RAGE null mice, and in the latter case we employed $\mathrm{Tg}$ mice with targeted expression of a DN form of the receptor in SMCs. Homozygous RAGE null mice display normal reproductive fitness, life span, and cardiovascular function in the 
absence of stress. In homeostasis, these animals display no obvious phenotype. Compared with wild-type mice, RAGE null mice did not display mRNA nor antigen for RAGE by either RT-PCR or Western blot analysis of lung tissue (Figure 3, a and b, respectively). Homozygous RAGE null mice backcrossed three generations into C57BL/6 displayed markedly decreased neointimal expansion compared with RAGE-bearing littermate controls on day 28 after injury (Figure 3c). Figures 3, d and $\mathrm{e}$, reveal representative micrographs from injured vessels on day 28 in wild-type littermates, compared with homozygous RAGE null mice, respectively. Importantly, consistent with a central role for preformed RAGE antigen in the endogenous response of the artery to acute injury, MPO activity of vessel segments retrieved from RAGE null mice was significantly lower than that observed in wild-type littermates 1 hour after injury (Figure 3f). No differences were observed in MPO levels between sham-treated RAGE null mice versus wild-type controls (data not shown). Importantly, although the levels of MPO activity in the RAGE null mouse were not reduced to those observed in sham-treated mice, these data, nevertheless suggest that, at least in part, endogenous levels of RAGE contribute to the innate response to acute vascular injury.

Because the immunohistochemistry studies in Figure 1 strongly suggested that the SMCs were the principal RAGE-expressing cell in the expanding neointima after injury, we directly tested the impact of RAGE signaling in SMCs on the response to arterial injury. We prepared $\mathrm{Tg}$ mice expressing a truncated form of RAGE devoid of the cytosolic domain (DN-RAGE). Although DN-RAGE bears the extracellular domain and transmembrane-spanning domain, the absence of the cytosolic domain renders the expressed protein devoid of RAGE signaling, even in cells bearing the wild-type receptor $(21,22,25)$. A transgene was created in which expression of DN-RAGE was placed under control of the SM22 $\alpha$ promoter (26) to direct expression to SMCs (termed Tg SM22-DN-RAGE). In these studies, transgenic mice were generated directly in the C57BL/6 background. Southern blot analysis revealed three Tg SM22-DN-RAGE founders (Figure 3g shows a typical founder). In our experiments, hemizygous $\mathrm{Tg}$

\section{Figure 3}

Role of RAGE in acute arterial injury: studies in mice with genetically manipulated levels of RAGE/RAGE function. (a-f) RAGE null mice. Lung tissue retrieved from homozygous RAGE null mice or wild-type animals was subjected to RT-PCR for detection of RAGE mRNA or Western blotting ( $\mathbf{a}$ and $\mathbf{b}$ ). Mice were subjected to femoral artery guide-wire injury. I/M ratio was determined on day 28 (c) and van Gieson's elastic staining was performed on a representative femoral artery section from a wild-type mouse (d) and a RAGE null mouse (e). Scale bar: $50 \mu \mathrm{m}$. (f) MPO activity. One hour after injury, vessel segments were retrieved from RAGE null and wild-type mice and subjected to MPO activity assays. Vessels were harvested and pooled from $n=2$ mice per condition. Data from three sets of pooled animals per condition are reported. ( $(\mathbf{-}-\mathbf{I})$ Tg SM22-DN-RAGE mice. Tg SM22-DN-RAGE mice were identified by Southern blotting $(\mathbf{g})$. RT-PCR was performed on samples from $\mathrm{Tg}$ mice overexpressing full-length RAGE (lanes 1 and 2) or DN-RAGE (lanes 3 and 4). (h) Western blotting was performed on lysates retrieved from the aortae of wild-type and $\mathrm{Tg}$ animals (i). Immunostaining with antiRAGE IgG of femoral artery from Tg SM22-DN-RAGE mice (k) compared with a vessel from a non-Tg littermate (j). Scale bar: $25 \mu \mathrm{m}$. (I) Tg SM22-DN-RAGE mice and littermates were subjected to femoral artery injury and $\mathrm{I} / \mathrm{M}$ ratio was determined on day 28 . In I, there were at least 10-20 vessels per experimental condition. $\mathbf{a}$
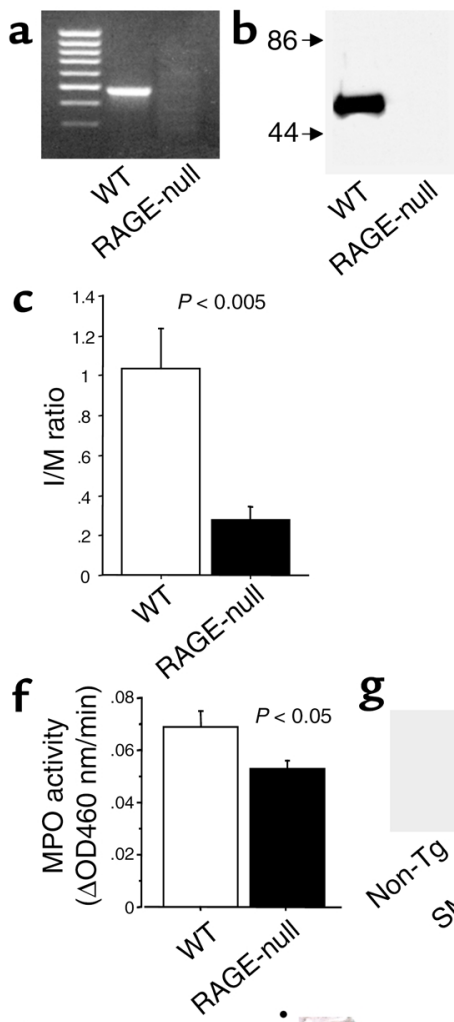

g
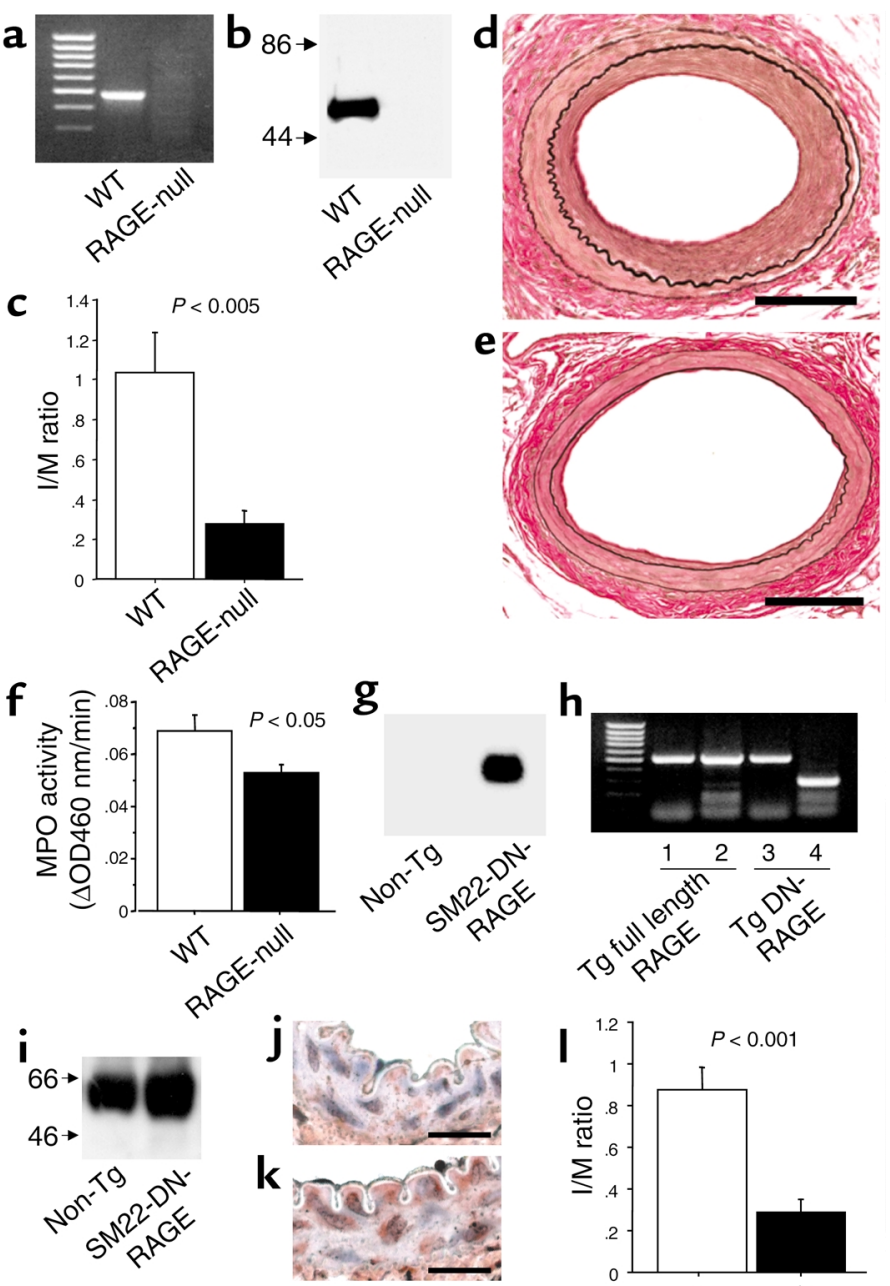

h
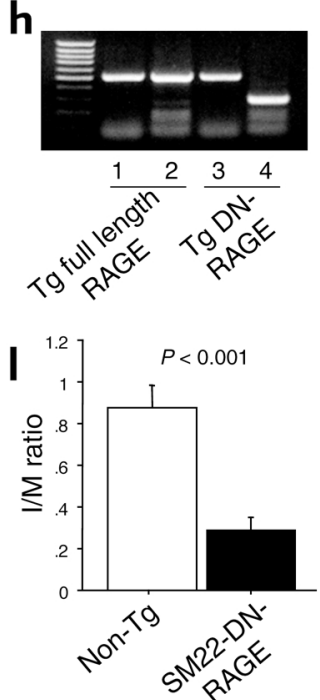

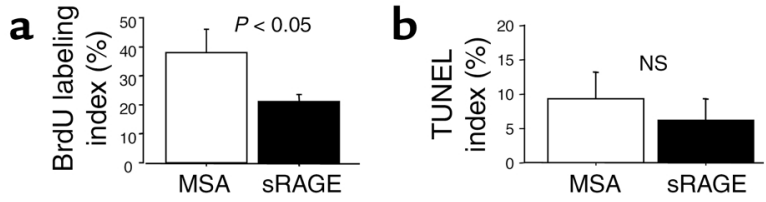

C

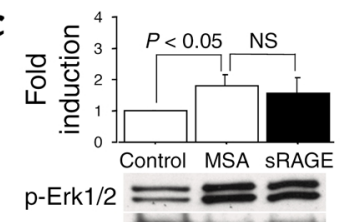

Total-Erk1/2

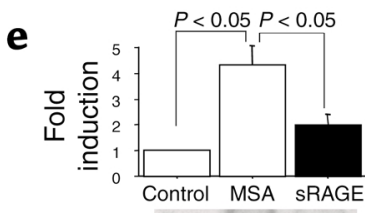

p-Jak2

Total-Jak2
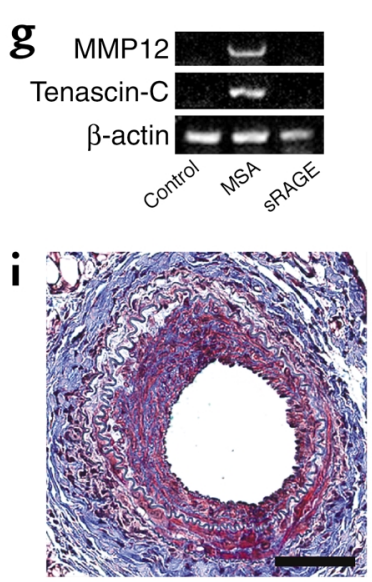

$f$
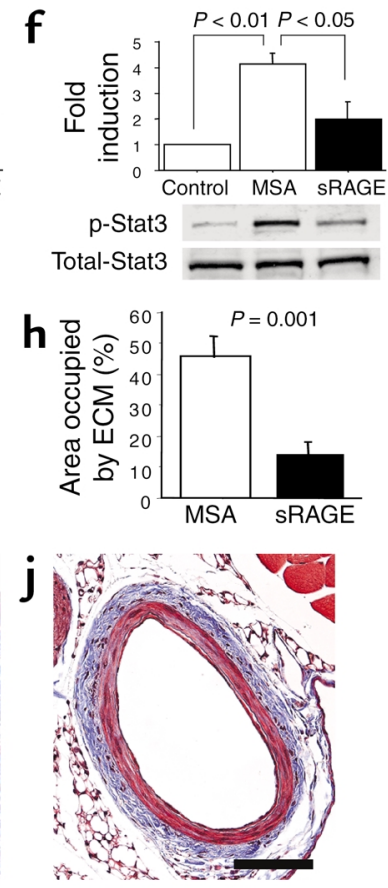

mice and littermate controls were employed. RT-PCR analysis was performed with primers spanning the region from the transmembrane-spanning domain to the poly A tail and demonstrated a smaller amplicon in samples from Tg SM22-DN-RAGE animals (Figure $3 \mathrm{~h}$, lane 4) compared with control mice overexpressing full-length RAGE (lane 2). This finding is consistent with deletion of the cytosolic tail of RAGE in transcripts from animals bearing the DN-RAGE transgene. In contrast, RT-PCR with primers spanning a portion of the extracellular domain of the receptor (which was identical in full-length and DN-RAGE transcripts) displayed an amplicon of the same size in the mice overexpressing full-length or DN-RAGE (Figure $3 \mathrm{~h}$, lanes 1 and 3 ). By Western blot analysis, the band immunoreactive with RAGE in lysates prepared from the aortae of Tg SM22-DN-RAGE mice was of greater intensity and breadth than that observed in wild-type mice (after normalization to levels of $\beta$-actin), consistent with the concept that DNRAGE would be expected to be slightly smaller than the wild-type receptor (Figure 3i). Consistent with

\section{Figure 4}

Mechanisms underlying sRAGE-mediated suppression of neointimal expansion in wild-type mice. (a) BrdU-labeling index. Mice were subjected to femoral artery guide-wire injury followed by intraperitoneal injection of BrdU and sacrifice on day 7. Nuclei immunoreactive with $\mathrm{BrdU}$ were quantitated, and the index in the neointima was reported. (b) TUNEL index. Mice were subjected to femoral artery guidewire injury followed by sacrifice on day 7 . Vessels were subjected to TUNEL staining, and data are expressed as an index of TUNEL-positive nuclei in the neointima. ( $c$ and $\mathbf{d}$ ) Activation of Erk1/2 and PKB. Mice were subjected to femoral artery guide-wire injury and sacrificed 30 min later. SDS-PAGE/Western blotting with Ab's to phospho-Erk1/2 (p-Erk1/2) or total Erk1/2 (c) or phospho-PKB (p-PKB) or total PKB were employed. (e and $\mathbf{f}$ ) Activation of Jak2/Stat3. Mice were subjected to femoral artery guide-wire injury and sacrificed 7 days later. SDS-PAGE/Western blotting with Ab's to phospho-Jak2 (p-Jak2) or total Jak2 (e) or phospho-Stat3 (p-Stat3) or total Stat3 (f) were employed. (g) Transcripts for MMP12, tenascin-C, and $\beta$-actin. Mice were subjected to femoral artery guide-wire injury. On day 7 , total RNA was harvested for RT-PCR using the indicated primers. (h, i, and $\mathbf{j})$ Masson's trichrome stain. Mice were subjected to femoral artery guide-wire injury and sacrificed on day 28. Sections of femoral artery were stained with Masson's trichrome reagent (i and $\mathbf{j}$ ), and images were analyzed to determine the area occupied by blue staining ECM (h). Scale bar: $50 \mu \mathrm{m}$. The above experiments employed at least eight vessels for each experimental condition, and experiments were repeated three times.

these observations, immunostaining of uninstrumented femoral arteries from Tg SM22-DN-RAGE mice demonstrated an increase in RAGE antigen in SMCs, presumably due to expression of the DN-RAGE transgene, compared with non- $\mathrm{Tg}$ littermates (Figure 3 , $\mathrm{k}$ and $\mathrm{j}$, respectively; note that RAGE antigen is expressed, albeit at a lower level, in the vascular SMCs of non-Tg mice). When Tg SM22-DN-RAGE mice were subjected to arterial injury, the resulting I/M ratio was significantly decreased compared with non-Tg littermates (Figure 31). Thus, these observations led us to focus our efforts on identifying the molecular mechanisms through which RAGE contributed to the response of vascular SMCs to arterial injury.

In vivo analyses of mechanisms underlying RAGE-dependent expansion of the neointima. First, we examined SMC proliferation in the injured artery in mice treated with sRAGE versus those mice treated with vehicle, MSA. In vivo BrdU labeling was performed on day 7 after acute arterial injury. A significant decrease in BrdU-labeling index was observed in sRAGE-treated mice compared with vehicle-treated controls after injury $(21 \% \pm 3 \%$ versus $38 \% \pm 8 \% ; P<0.05$; Figure $4 a$ ). In contrast, few BrdU-labeled cells were evident in the media at this time point (data not shown). Because of the possibility that decreased neointimal expansion might also reflect cell loss through apoptosis, evidence of cellular commitment to programmed cell death was sought. Thus, vascular sections were studied using the TUNEL method. However, TUNEL-positive nuclei were detected with very low frequency in both control and sRAGEtreated vessel segments 7 days after injury (Figure 4b). 
Similarly, a paucity of TUNEL-positive nuclei was observed in sections from sRAGE-treated mice at earlier and later time points (data not shown). These data are consistent with previous work in this model demonstrating that only at 14 days after injury was there any evidence of significant apoptosis in the neointima (41). Since the impact of blockade of RAGE was only evident when sRAGE was administered in the first 7 days after injury, these findings support the con- cept that RAGE blockade partially suppressed intimal proliferation, without increasing apoptosis in the injured arterial wall.

Next, we sought to examine the RAGE-dependent signal transduction pathways linked to cellular proliferation after arterial injury. Previous studies have shown that the mitogen-activated protein kinase pathway, especially extracellular signal-regulated protein kinase (Erk1/2) and PI3K, undergo activation in the early
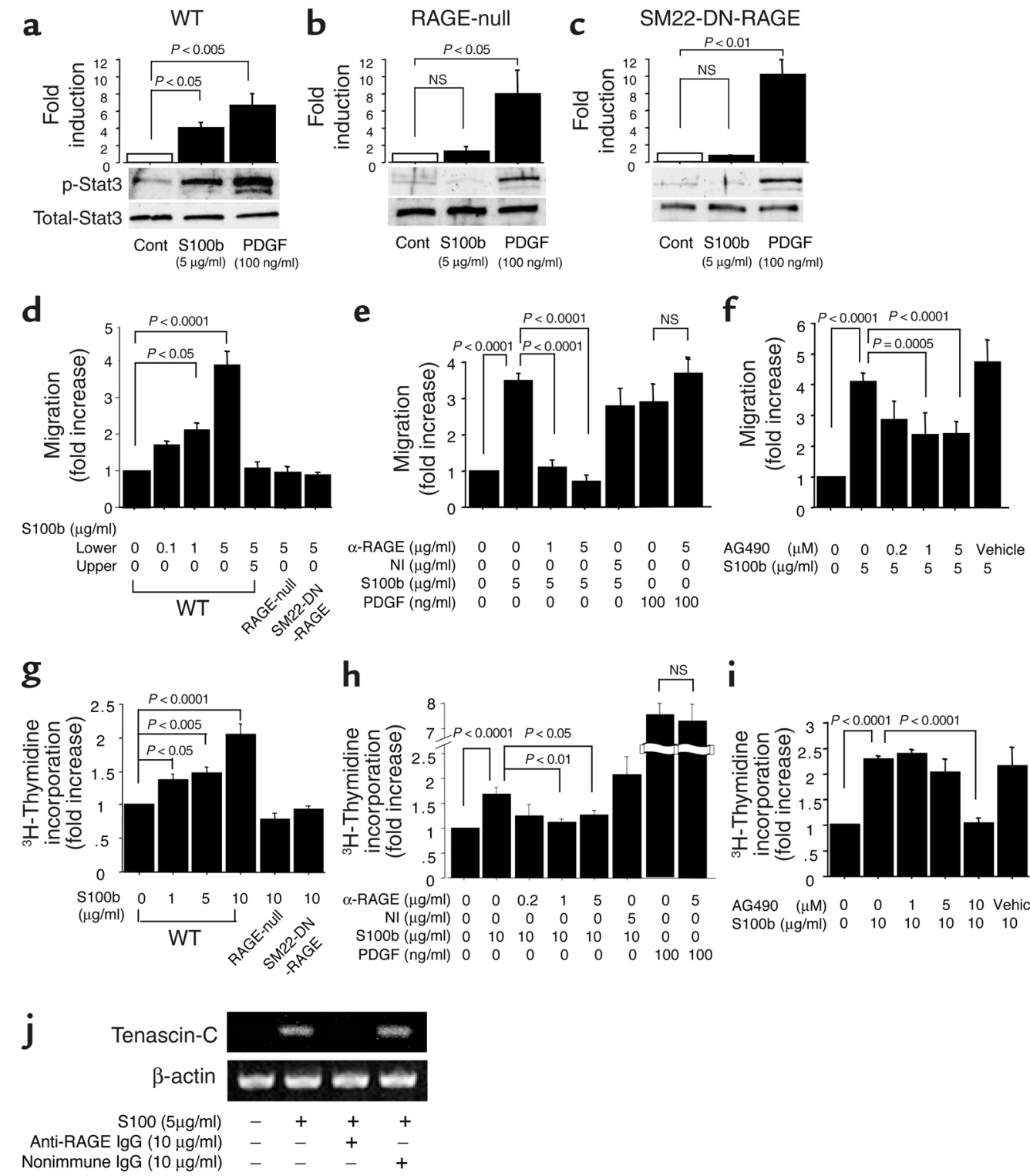

Figure 5

Effect of RAGE on properties of cultured SMCs. (a-c) Aortic SMCs from wild-type (a), RAGE null (b), and Tg SM22-DN-RAGE mice (c) were exposed to S100b or PDGF for 10 min, and cells were harvested for phospho/total-Stat3 by Western blotting. (d-f) Cultured SMCs were added to the upper compartment of microchemotaxis chambers, and S100b was added to upper/lower compartment for $4 \mathrm{~h}$ (d). The migration assay was performed as in $\mathbf{d}$, except that cells were preincubated with anti-RAGE IgG or nonimmune IgG. Migration was initiated by addition of either S100b or PDGF (lower compartment) (e). The migration of wild-type SMCs preincubated for 30 min with AG490 was studied as in d in the presence of S100b $(5 \mu \mathrm{g} / \mathrm{ml})(\mathbf{f})$. (g-i) Serum-starved cultured SMCs from wild-type mice were incubated with the S100b for 2 days, and DNA synthesis was quantitated by ${ }^{3} \mathrm{H}$-incorporation (g). SMCs cultured from RAGE null or Tg SM22-DN-RAGE mice were exposed to S100b or PDGF (g). The assay was performed as in $\mathbf{g}$ (wild-type SMCs), except that anti-RAGE IgG or nonimmune IgG was added, and cultures were exposed to S100b or PDGF (h). Cultured SMCs from wild-type mice were exposed to $\mathrm{S} 100 \mathrm{~b}$ in the presence of $\mathrm{AG} 490$, and ${ }^{3} \mathrm{H}$-thymidine incorporation was determined (i). (j) SMCs from wild-type mice were exposed to S100b for $12 \mathrm{~h}$, and RNA was harvested for RT-PCR using primers for tenascin-C or $\beta$-actin. 

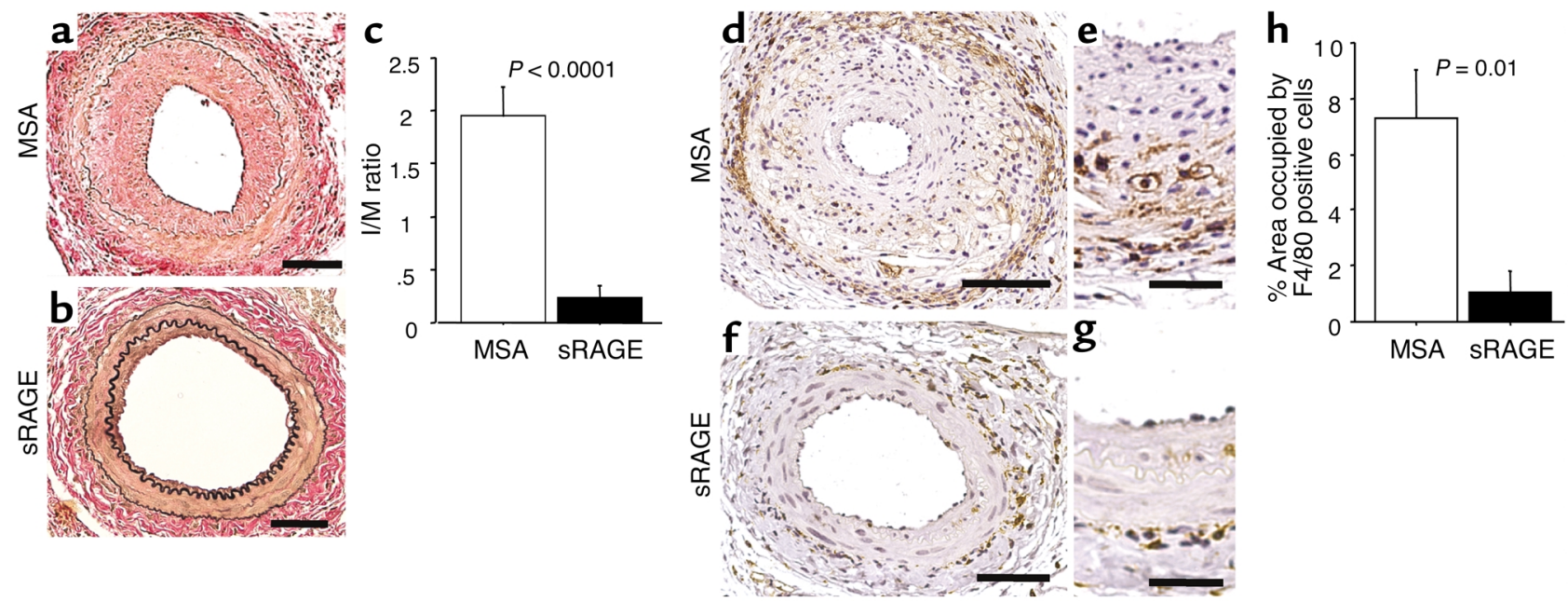

\section{Figure 6}

Effect of sRAGE on neointimal expansion in apoE null mice. (a-h) ApoE null mice were subjected to femoral artery guide-wire injury (day 1 ), and animals were treated with either sRAGE or MSA $(100 \mu \mathrm{g} /$ day, intraperitoneally, days $0-7)$ and sacrificed on day 28 . Representative sections were treated with van Gieson's elastic stain ( $\mathbf{a}$ and $\mathbf{b})$ or subjected to immunohistochemistry with anti-F4/80 IgG ( $\mathbf{d}-\mathbf{g})$. The l/M ratio was determined on day 28 in the two groups of mice (c). (h) For 4/80-stained sections, image analysis was performed, and the area occupied by immunoreactive cells in the neointima and media was determined. These experiments employed at least eight vessels for each experimental condition, and the experiment was repeated twice. Scale bars: $\mathbf{a}, \mathbf{b}, \mathbf{d}$, and $\mathbf{f}, 50 \mu \mathrm{m}$, and $\mathbf{e}$ and $\mathbf{g}, 25 \mu \mathrm{m}$.

phase of cellular proliferation in the repairing arterial segment $(42,43)$. To study these cascades, phosphorylation of Erk1/2 and protein kinase B (PKB), the latter a downstream target of PI3K (44), was assessed. Homogenates of the damaged artery were harvested 30 min after the injury. Immunoblot analysis revealed increased levels of phospho-Erk1/2 and phospho-PKB, compared with uninstrumented arterial segments (approximately 1.75 - and 2.5 -fold, respectively; $P<0.05$ ) (Figure 4, $c$ and d). In mice treated with sRAGE, however, no suppression of phosphorylation of Erk $1 / 2$ or PKB was observed (Figure 4, $c$ and d). Previous work has shown that pharmacologic blockade of mitogenactivated protein kinase-1 or PI3K suppressed an early phase of medial cell proliferation, but was without effect on intimal proliferation, the latter contributing centrally to restenosis in rodent models $(42,43)$. A recent study, however, demonstrated activation of Jak2 and Stat 3 (as well as more transient activation of Stat1) following arterial injury (45). In support of a role for Jak2 in these pathways, it has been shown that the Jak2 antagonist AG490 suppressed neointimal SMC replication and reduced neointimal formation after arterial injury (45). Because RAGE-mediated activation of the JAK/STAT pathway has been shown previously in a line of cultured cells (46), it was therefore logical to test the impact of RAGE blockade on this pathway in vivo. Thus, we analyzed phosphorylation of Jak2 and Stat3 in injured femoral artery segments. On day 7 , increased phospho-Jak2 and phospho-Stat 3 were observed, compared with untreated controls (approximately fourfold), consistent with activation of these signaling proteins (Figure 4, e and f). Arterial segments retrieved on day 7 from animals treated with sRAGE displayed significantly decreased suppression of Jak2 and Stat3 phosphorylation $(P<0.05$; Figure 4 , e and $\mathrm{f})$. These findings suggested that RAGE-dependent phosphorylation of Jak2 and Stat 3 contributes to enhanced SMC proliferation in the acutely injured vessel wall.

Neointimal expansion in the injured vessel wall depends on a number of factors beyond increased cellular proliferation, such as remodeling and expansion of the ECM (47). A recent study in the rat carotid balloon angioplasty model demonstrated four temporal patterns of altered gene expression compared with uninjured vessels (48). One pattern (wave 3), involving expression of tenascin-C and MMP12, both linked to accumulation and remodeling of ECM, was especially striking; in each case, gene expression was strongly and continuously increased until day 28. Transcripts for tenascin-C and MMP12 were evident on day 7 after vascular injury compared with samples from uninjured control vessels by RT-PCR (Figure 4g). In animals treated with sRAGE, however, suppression of transcripts for both tenascin-C and MMP12 were observed. Induction of each of these genes was confirmed at both time points in MSA-treated controls, whereas administration of sRAGE led to significant reduction in transcript levels for each (Figure 4g). To assess overall accumulation of ECM after femoral artery injury, Masson's trichrome staining on vessels harvested on day 28 was employed. Compared with uninjured controls, a representative femoral artery from an injured vessel displayed neointimal expansion and evidence of increased ECM (blue-staining material) (Figure 4, i and h). Both neointimal expansion and ECM were decreased by treatment with sRAGE, compared with MSA-treated animals (Figure 4, j and i, respectively and Figure 4h). 
In vitro analyses of mechanisms underlying RAGE-dependent expansion of the neointima. First, it was necessary to confirm RAGE-dependent activation of the JAK/STAT pathway in SMCs retrieved from mouse aorta. RAGE antigen was present on cultured SMCs from wild-type and Tg SM22-DN-RAGE mice, but not on cells from RAGE null mice. To test these concepts, aortic SMCs were incubated with the RAGE ligand S100b (22), and cellular properties were examined. When SMC cultures from wildtype mice were exposed to S100b, phosphorylation of Stat 3 was evident (Figure 5a), consistent with our observations in injured vessel segments in vivo (Figure 4f). SMCs retrieved from the aorta of RAGE null mice and Tg SM22-DN-RAGE animals, however, failed to reveal S100b-stimulated phosphorylation of Stat3 (Figure 5, b and $c$, respectively), consistent with studies in injured arterial segments. In contrast, using a different stimulus, PDGF, which is not a ligand for RAGE, no suppression of phosphorylation of Stat3 was observed in cultured SMCs from wild-type, RAGE null, or Tg SM22-DNRAGE animals (Figure 5, a-c, respectively). These data support the contention that ligand-RAGE interaction is a distinct axis resulting in Stat3 activation in SMCs.

In addition to cellular proliferation and excess ECM formation/deposition after arterial injury, we studied the role of SMC RAGE in mediating cellular migration, a key facet of neointimal expansion after injury $(8,47)$. Indeed, previous studies demonstrated key roles for RAGE in mediating ligand-stimulated migration of RAGE-bearing MPs and tumor cells $(20,21)$. Here, we first assessed the impact of this axis on SMCs using the RAGE ligand $\mathrm{S} 100 \mathrm{~b}$ as a chemotactic stimulus. In modified Boyden chambers, addition of increasing amounts of S100b to the lower compartment increased the number of migrating SMCs retrieved from the aortae of wild-type mice in a dose-dependent manner (Figure 5d). Importantly, cell migration was directional, i.e., not simply due to chemokinesis, because simultaneous placement of S100b in the upper compartment, thus blocking concentration gradient initiated by RAGE ligand in the lower compartment, decreased SMC movement (Figure 5d). Evidence for the RAGE dependence of these phenomena was shown using RAGE-modified mice. S100b-induced migration of SMCs was markedly decreased in cells retrieved from RAGE null and Tg SM22-DN-RAGE mice (Figure 5d), although their chemotaxis to PDGF was not impaired (data not shown), consistent with the concept that the latter was not a ligand for RAGE. Furthermore, chemotaxis of SMC retrieved and cultured from wild-type mice induced by $\mathrm{S} 100 \mathrm{~B}$ was decreased in the presence of anti-RAGE IgG, but not by nonimmune IgG (Figure 5e). RAGE blockade had no effect, however, on chemotaxis mediated by PDGF (Figure 5e). An important role for the JAK/STAT pathway in S100b-induced SMC migration was consistent with the partial inhibitory effect of AG490 (Figure 5f).

Next, we assessed the impact of RAGE-dependent SMC proliferation using ${ }^{3} \mathrm{H}$-thymidine incorporation as the biologic end point for these experiments. Incubation of SMCs from wild-type mice with S100b increased incorporation of ${ }^{3} \mathrm{H}$-thymidine, with a maximum of approximately twofold (Figure $5 \mathrm{~g}$; the increase in incorporation of ${ }^{3} \mathrm{H}$-thymidine correlated with an increase in SMC number). SMC proliferation in response to $S 100 \mathrm{~b}$ was significantly blunted in cells retrieved from RAGE null or Tg SM22-DN-RAGE animals (Figure 5g), but was not affected in response to PDGF (data not shown). Furthermore, SMC incubation with anti-RAGE IgG suppressed incorporation of ${ }^{3} \mathrm{H}$-thymidine in response to $\mathrm{S} 100 \mathrm{~b}$, but nonimmune IgG had no effect (Figure 5h). Incubation of wild-type SMCs with S100b in the presence of AG490 resulted in a dose-dependent suppression of SMC proliferation (down to baseline levels) (Figure 5i). These findings were consistent with our in vivo studies and strongly suggested that RAGE-dependent mechanisms were linked to phosphorylation of Stat 3 and cellular proliferation, in parallel.

Finally, we sought to test the impact of RAGE activation by S100b in SMCs on expression of tenascin-C. When SMCs from wild-type mice were exposed to S100b, RT-PCR analysis demonstrated upregulation of tenascin-C transcripts (Figure 5j). Upregulation of tenascin-C transcripts by $\mathrm{S} 100 \mathrm{~b}$ was blocked in the presence of anti-RAGE IgG, but not by equal concentrations of nonimmune IgG (Figure 5j).

Taken together, these studies employing SMCs retrieved directly from wild-type mice as well as RAGE-modified mice revealed that RAGE-dependent stimulation by S100b impacted importantly on SMC proliferation, migration, and expression of tenascin-C, all key features linked to neointimal expansion after arterial injury.

RAGE-dependent suppression of neointimal expansion in an atherosclerosis-prone environment - studies in apoE null mice. Lastly, we sought to test the impact of RAGE in neointimal expansion in an environment more likely to mirror that observed in the primate vessel wall in which hyperlipidemia importantly modulates the response to arterial injury. To simulate an atherosclerosis-prone environment, we induced femoral artery endothelial denudation injury in apoE null mice and assessed the impact of RAGE blockade. We employed apoE null mice in the C57BL/ 6 background at age 12 weeks, a time at which frank atherosclerosis in the femoral arteries has not yet occurred to a measurable degree, though hypercholesterolemia is certainly present (49). Our past studies indicated that although administration of sRAGE impacted on initiation and progression of atherosclerosis in these animals, there was no effect on the levels/profile of lipid $(17,18)$. Upon femoral artery injury, animals treated with sRAGE from day 0 through day 7 (injury on day 1) demonstrated attenuation of neointima with an $\mathrm{I} / \mathrm{M}$ ratio of $0.21 \pm 0.11$ versus that observed in MSA-treated mice, $1.93 \pm 0.35$ $(P<0.0001$; Figure 6, a-c). Furthermore, in marked contrast to analyses in wild-type C57BL/6 mice, in 
which few MPs are observed in the neointima/media of injured arterial vessels (28), histologic analysis demonstrated the presence of infiltrating MPs in the expanding neointima of apoE null mice treated with MSA at 28 days (Figure 6, d, e, h). In apoE null mice treated with sRAGE, however, a prominent decrease in numbers of MPs present in the neointima and media after injury, as demonstrated by immunohistochemistry using anti-F4/80 IgG, was observed (Figure 6, f-h).

\section{Discussion}

Mechanisms underlying neointimal expansion triggered by denuding arterial injury span a broad range of fundamental biologic pathways, as diverse as proteolytic activity capable of cleaving insulin-like growth factor binding protein-4 (50), vessel wall expression of chemokines $(51,52)$, and the well-established contributions of growth factors for SMCs $(8,53,54)$. A key facet of the biology of restenosis is that mechanisms linked to reprogramming of vascular properties are set and well underway within the first hours and days after injury; once triggered, such pathways lead to neointimal expansion over the ensuing days to weeks. Here, we have shown that RAGE-dependent mechanisms in modulation of SMC properties are engaged within the first hours to days after injury; indeed, administration of sRAGE was ineffective when first initiated beyond the 7 days immediately following acute arterial injury. Although it is difficult to be certain which occurs first, ligand accumulation or upregulated receptor expression, based on studies presented here we posit the following likely scenario. We speculate that enhanced MPO activity within the first hour after injury, induced at least in part by polymorphonuclear leukocytes attracted immediately to the injured arterial segment, triggers oxidant stress (39), thereby providing a definitive mechanism leading to formation of AGEs (38-40). We hypothesize that once formed, AGE ligands of the receptor likely stimulate enhanced expression of RAGE itself $(55,56)$. In parallel, studies have suggested that acute injury, as well as proinflammatory stimuli, may enhance expression of S100/calgranulin ligands of RAGE (34-37), thus providing a mechanism for enhanced RAGE expression, as well. Indeed, S100/calgranulins are expressed in a wide range of cell types, including SMCs and fibroblasts (57-59) and, in fact, may be released from activated cells, thereby facilitating interaction with cell surface RAGE (60). Our immunohistochemical studies, as displayed in Figure 1, strongly suggest that $\mathrm{S} 100$ s may be expressed in injured SMCs and adventitial cells. Taken together with increased accumulation/formation of AGEs in the injured vascular segment, we propose that enhanced ligand-receptor expression provides a novel axis leading to sustained cellular activation and stress, and, thus, exaggerated neointimal formation after acute arterial injury.

Yet, the situation appears likely to be more complex. Specifically, endogenous very low levels of RAGE antigen in the artery subjected to injury likely contribute, at least in part, to the vascular response. As RAGE null mice displayed decreased MPO activity within $1 \mathrm{~h}$ after denuding arterial injury compared with wild-type animals, these findings prompt the hypothesis that RAGE plays an innate and immediate role in the response to arterial injury. Furthermore, upon enhanced injury-triggered expression/formation of ligands such as AGEs and S100/calgranulins, RAGE expression is further augmented, thereby importantly modulating the vessel wall response. RAGE-dependent mechanisms, at least in part, then contribute to increased SMC migration, proliferation, and expression of ECM proteins and, thus, exaggerated neointimal formation and restenosis.

Importantly, it is essential to note that in our in vitro analyses, blockade/genetic deletion or modification of SMC RAGE did not affect the SMC response to PDGF in that RAGE blockade had no effect on SMC proliferation or migration in the presence of PDGF (Figure 5). These findings highlight the concept that RAGE is engaged and activated by distinct ligands within the injured vessel wall and that PDGF is not one such ligand. Although it is highly possible that levels of PDGF are reduced in RAGE-blocked arterial segments in vivo, however, the means by which this would occur is not dependent on direct stimulation of RAGE. Rather, it is possible that in vivo, blockade of the ligand-RAGE axis may impact on PDGF generation in the injured environment by interception of common signalling pathways downstream of the initiating cell surface events.

It is well-recognized that the vascular milieu of wildtype mice is quite different from that in an atherosclerosis-prone environment. It was thus for this reason that we performed studies in apoE null mice. In apoE null mice, the response to arterial injury involves inflammatory mechanisms associated with an influx of MPs (compared with wild-type animals), as well as proliferative/migratory SMC components linked importantly to the vascular response to acute injury $(8,15,16$, $47,52,61-65)$. In the present studies, in parallel with diminished neointimal expansion after injury, numbers of MPs infiltrating the injured vessel wall were strikingly reduced (Figure 6). These findings are analogous to the decrease in numbers of MPs (and SMCs) infiltrating established atherosclerotic plaques in diabetic apoE null mice treated with sRAGE $(17,18)$. In that setting, analogous to that after acute arterial injury, atherosclerotic plaques are enriched in the same two ligands of RAGE, AGEs, and S100/calgranulins $(17,18,66)$. Diabetic apoE null mice developed more severe atherosclerosis in the initial stages, as well as displayed increased progression of established atherosclerosis, compared with nondiabetic, apoE null animals. Administration of sRAGE decreased lesion area and complexity in parallel with reduced numbers of MPs and SMCs $(17,18,66)$, at least in part, mediated by decreased vascular expression of vascular cell adhesion molecule- 1 and JE-MCP-1 $(18,66)$. In view of the likely central role of JE-MCP-1 in atherosclerosis, a preliminary report from our laboratory demonstrated that 
this chemokine was generated in cultured SMCs stimulated by AGEs (67). Thus, it is highly likely that JEMCP-1, in part, augments MP migration into the injured vessel wall of apoE null mice. To rigorously test these concepts, ongoing studies are focused on introduction of DN-RAGE in either SMCs or MPs in apoE null mice, the latter driven by the macrophage scavenger receptor promoter (68).

Taken together, these findings highlight the concept that RAGE is a logical and directed target for effective therapeutic intervention in two settings germane to primate vascular biology, that is, in innate chronic atherosclerosis as well as acute vascular injury. These findings highlight critical and highly relevant distinctions in current vascular-protective strategies. Although sirolimus coating of stents provided by far the most optimal suppression of restenosis in animals as well as humans, the impact of this agent on the adaptive immune response sharply limits its potential for systemic administration in long-standing chronic atherosclerosis.

In striking contrast, experimental evidence suggests that blockade of RAGE provides a means to limit untoward vascular responses to both chronic and acute injury within the vessel wall. Although differences certainly exist between the reparative response to denuding arterial injury in rodents versus higher species such as pigs and primates (47), we conclude that our findings implicate fundamental roles for RAGE in the MP and SMC response to arterial injury and highlight the rationale for testing the impact of blockade of this axis as a means to limit untoward neointimal expansion in humans.

\section{Acknowledgments}

This work was supported by grants from the NIH (KO8 HL04484), the Juvenile Diabetes Research Foundation, United States Public Health Service (HL60901), Uehara Memorial Foundation, and the Surgical and Cardiothoracic Research Fund. P.P. Nawroth was supported by grants from the Deutsche Forschungsgemeinschaft. A.M. Schmidt is a recipient of a Burroughs Wellcome Fund Clinical Scientist Award in Translational Research. Yoshifumi Naka is the Herbert Irving Assistant Professor of Surgery.

1. Bittl, J. 1996. Advances in coronary angioplasty. N. Engl. J. Med. 24:1290-1302.

2. Knight, C.J., et al. 1999. Stent implantation reduces restenosis in patients with suboptimal results following coronary angioplasty. Eur. Heart J. 20:1783-1790.

3. Babapulle, M.N., and Eisenberg, M.J. 2002. Coated stents for the prevention of restenosis. Part I. Circulation. 106:2734-2740.

4. Babapulle, M.N., and Eisenberg, M.J. 2002. Coated stents for the prevention of restenosis. Part II. Circulation. 106:2859-2866.

5. Suzuki, T., et al. 2001. Stent-based delivery of sirolimus reduces neointimal formation in a porcine coronary model. Circulation. 104:1188-1193.

6. Klugherz, B.D., et al. 2002. Twenty eight day efficacy and pharmacokinetics of the sirolimus-eluting stent. Coron. Artery Dis. 13:183-188.

7. Morice, M.C., et al. 2002. A randomized comparison of a sirolimus-eluting stent with a standard stent for coronary revascularization. N. Engl.J. Med. 346:1773-1780.

8. Schwartz, S., Reidy, M., and O’Brien, E. 1995. Assessment of factors important in atherosclerotic occlusion and restenosis. Thromb. Haemost. 74:541-551.

9. Ross, R. 1999. Atherosclerosis an inflammatory disease. N. Engl. J. Med. 340:115-126.
10. Yamamoto, K., et al. 2000. Ribozyme oligonucleotides against transforming growth factor-beta inhibit neointimal formation after vascular injury in a rat model. Circulation. 102:1308-1314.

11. Popma, J., Califf, R., and Topol, E. 1991. Clinical trials of restenosis after coronary angioplasty. Circulation. 84:1426-1436.

12. Liu, M., Roubin, G., and King, S. 1999. Trapidil in preventing restenosis after balloon angioplasty in the atherosclerotic rabbit. Circulation. 79:1374-1388.

13. Majesky, M.W., Lindner, V., Twardzik, D.R., Schwartz, S.M., and Reidy, M.A. 1991. Production of transforming growth factor beta 1 during repair of arterial injury. J. Clin. Invest. 88:904-910.

14. Nikol, S., et al. 1992. Expression of transforming growth factor-beta 1 is increased in human vascular restenosis lesions. J. Clin. Invest. 90:582-1592.

15. Danenberg, H.D., et al. 2002. Macrophage depletion by clodronate-containing liposomes reduces neointimal formation after balloon injury in rats and rabbits. Circulation. 106:599-605.

16. Egashira, K., et al. 2002. Importance of monocyte chemoattractant protein-1 pathway in neointimal hyperplasia after periarterial injury in mice and monkeys. Circ. Res. 90:1167-1172.

17. Park, L., et al. 1998. Suppression of accelerated diabetic atherosclerosis by soluble receptor for AGE (sRAGE). Nat. Med. 4:1025-1031.

18. Bucciarelli, L.G., et al. 2002. RAGE blockade stabilizes established atherosclerosis in diabetic apolipoprotein E null mice. Circulation. 106:2827-2835.

19. Wendt, T., et al. 2002. Receptor for advanced glycation endproducts (RAGE) and vascular inflammation: insights into the pathogenesis of macrovascular complications in diabetes. Curr. Atheroscler. Rep. 4:228-237.

20. Schmidt, A.M., et al. 1993. Regulation of human mononuclear phagocyte migration by cell surface binding proteins for AGEs. J. Clin. Invest. 91:2155-2168

21. Taguchi, A., et al. 2000. Blockade of RAGE/amphoterin suppresses tumor growth and metastases. Nature. 405:354-360.

22. Hofmann, M.A., et al. 1999. RAGE mediates a novel proinflammatory axis: the cell surface receptor for S100/calgranulin polypeptides. Cell. 97:889-901.

23. Yan, S.D., et al. 1997. Amyloid- $\beta$ peptide-RAGE interaction elicits neuronal expression of M-CSF: a proinflammatory pathway in Alzheimer's disease. Proc. Natl. Acad. Sci. U. S. A. 94:5296-5301.

24. Schmidt, A.M., et al. 1994. RAGE has a central role in vessel wall interactions and gene activation in response to AGEs. Proc. Natl. Acad. Sci. U. S. A. 91:8807-8811.

25. Kislinger, T., et al. 1999. N(epsilon)-(carboxymethyl)lysine adducts of proteins are ligands for RAGE that activate cell signalling pathways and modulate gene expression. J. Biol. Chem. 274:31740-31749.

26. Li, L., Miano, J.M., Mercer, B., and Olson, E.N. 1996. Expression of the SM22 alpha promoter in transgenic mice provides evidence for distinct transcriptional regulatory programs in vascular and visceral smooth muscle cells. J. Cell. Biol. 132:849-859.

27. Zhang, S.H, Reddick, R.L., Piedrahita, J.A., and Maeda, N. 1992. Spontaneous hypercholesterolemia and arterial lesions in mice lacking apolipoprotein E. Science. 258:468-471.

28. Roque, M., et al. 2000. Mouse model of femoral artery denudation injury associated with the rapid accumulation of adhesion molecules on the luminal surface and recruitment of neutrophils. Arterioscler. Thromb. Vasc. Biol. 20:335-342.

29. Yan, S.D., et al. 1995. Nonenzymatically glycated tau in Alzheimer's disease induces neuronal oxidant stress resulting in cytokine gene expression and release of A $\beta$. Nat. Med. 1:693-699.

30. Johnson, D., Gautsch, J., Sportsman, J., and Elder, J. 1984. Improved technique utilizing non-fat dry milk for analysis of proteins and nucleic acids transferred to nitrocellulose. Gene. Anal. Tech. 1:3-8.

31. Goldblum, S.E., Wu, K.M., and Jay, M. 1985. Lung myeloperoxidase as a measure of pulmonary leukostasis in rabbits. J. Appl. Physiol. 59:1978-1985.

32. Tarvo, P., and Barret, G. 1980. Differences in proliferation of primary cultures of vascular smooth muscle cells taken from male and female rats. Blood Vessels. 17:110-116.

33. Roque, M., et al. 2001. Effect of p27 deficiency and rapamycin on intimal hyperplasia: in vivo and in vitro studies using a p27 knockout mouse model. Lab. Invest. 81:895-903.

34. Thorey, I.S., et al. 2001. The $\mathrm{Ca}^{2+}$-binding proteins S100A8 and S100A9 are encoded by novel injury-related genes. J. Biol. Chem. 276:35818-35825.

35. Hu, S.P., Harrison, C., Xu, K., Cornish, C.J., and Geczy, C.L. 1996. Induction of the chemotactic S100 protein, CP-10, in monocyte/macrophages by lipopolysaccharide. Blood. 87:3919-3928.

36. Xu, K., and Geczy, C.L. 2000. IFN-gamma and TNF regulate macrophage expression of the chemotactic S100 protein S100A8. J. Immunol. 164:4916-4923.

37. Xu, K., Yen, T., and Geczy, C.L. 2001. IL-10 upregulates macrophage expression of the S100 protein S100A8. J. Immunol. 166:6358-6366. 
38. Miyata, T., et al. 1999. OPB-9195 treatment inhibits intimal thickening balloon injury of rat carotid artery: role of glycoxidation and lipoxidation reactions in vascular tissue damage. FEBS Lett. 445:202-206.

39. Jansson, G. 1991. Oestrogen-induced enhancement of myeloperoxidase activity in polymorphonuclear leukocytes-a possible cause of oxidative stress in inflammatory cells. Free Radic. Res. Commun. 14:195-208.

40. Anderson, M.M., Requena, J.R., Crowley, J.R., Thorpe, S.R., and Heinecke, J. 1999. The myeloperoxidase system of human phagocytes generates Nepsilon-(carboxymethyl)lysine on proteins: a mechanism for producing advanced glycation endproducts at sites of inflammation. J. Clin. Invest. 104:103-113.

41. Reis, E.D., et al. 2000. Apoptosis, proliferation and p27 expression during vessel wall healing: time course study in a mouse model of transluminal femoral artery injury. J. Vasc. Surg. 32:1022-1029.

42. Koyama, H., Olson, N., Dastva, F., and Reidy, M. 1998. Cell replication in the arterial wall. Circ. Res. 82:713-721.

43. Shigematsu, K., Koyama, H., Olson, N., Cho, A., and Reidy, M. 2000. Phosphatidylinositiol 3-kinase signaling is important for smooth muscle cell replication after arterial injury. Arterioscler. Thromb. Vasc. Biol. 20:2373-2378.

44. Carpenter, C., and Cantley, L. 1996. Phosphoinositide kinases. Curr. Opin. Cell. Biol. 8:153-158.

45. Seki, Y., et al. 2000. Role of the JAK/STAT pathway in rat carotid artery remodeling after vascular injury. Circ. Res. 87:12-18.

46. Huang, J.S., et al. 2001. Role of RAGE and the JAK/STAT-signalling pathway in AGE-induced collagen production in NRK-49F cells. J. Cell. Biochem. 81:102-113.

47. Schwartz, R., Homes, D., and Topol, E. 1992. The restenosis paradigm revisited; an alternative proposal for cellular mechanisms. J. Am. Coll. Cardiol. 20:1284-1293.

48. Tai, J.T., et al. 2000. Determination of temporal expression for multiple genes in the rat carotid artery injury model. Arterioscler. Thromb. Vasc. Biol. 20:2184-2191.

49. Nakashima, Y., Plump, A., Raines, E., Breslow, J., and Ross, R. 1994. ApoE-deficient mice develop lesions of all phases of atherosclerosis throughout the arterial wall. Arterioscler. Thromb. 141:133-140.

50. Bayes-Genis, A., et al. 2001. Insulin-like growth factor binding protein-4 protease produced by smooth muscle cells increases in the coronary artery after angioplasty. Arterioscler. Thromb. Vasc. Biol. 21:335-341.

51. Furukawa, Y., et al. 1999. Anti-MCP-1/MCAF antibody inhibits neointimal hyperplasia in injured rat carotid arteries. Circ. Res. 84:306-314.

52. Reis, E.D., et al. 2000. Sulindac inhibits neointimal formation after arterial injury in wild-type and apolipoprotein-E deficient mice. Proc. Natl. Acad. Sci. U. S. A. 97:12764-12769.

53. Majesky, M.W., et al. 1990. PDGF ligand and receptor gene expression during repair of arterial injury. J. Cell Biol. 111:2149-2158.
54. Daemen, J., Lombardi, D., Bosman, F., and Schwartz, S. 1991. Angiotensin II induces smooth muscle proliferation in the normal and injured rat arterial wall. Circ. Res. 68:450-456.

55. Li, J., Qu, X., and Schmidt, A.M. 1998. Sp1 binding elements in the promoter of RAGE are essential for amphoterin-mediated gene expression in cultured neuroblastoma cells. J. Biol. Chem. 273:30870-30878.

56. Li, J., and Schmidt, A.M. 1997. Characterization and functional analysis of the promoter of RAGE. J. Biol. Chem. 272:16498-16506.

57. Zimmer, D.B., Cornwall, E.H., Landar, A., and Song, W. 1995. The S100 protein family: history, function, and expression. Brain Res. Bull. 37:417-429.

58. Schafer, B.W., and Heinzmann, C.W. 1996. The S100 family of EF-hand calcium-binding proteins: functions and pathology. TIBS. 21:134-140.

59. Mandinova, A., et al. 1998. Distinct subcellular localization of calcium binding S100 proteins in human smooth muscle cells and their relocation in response to rises in intracellular calcium. J. Cell Sci. 111:2043-2054

60. Rammes, A., et al. 1997. Myeloid-related protein (MRP) 8 and MRP14, calcium-binding proteins of the S100 family, are secreted by activated monocytes via a novel, tubulin-dependent pathway. J. Biol. Chem. 272:9496-9502.

61. Rogers, C., Welt, F., Karnovsky, M., and Edelman, E. 1996. Monocyte recruitment and neointimal hyperplasia in rabbits: coupled inhibitory effects of heparin. Arterioscler. Thromb. Vasc. Biol. 16:1312-1318.

62. Rogers, C., Edelman, E.R., and Simon, D.I. 1998. An antibody to the $\beta 2$-leukocyte integrin Mac-2 reduces intimal thickening after angioplasty or stent implantation in rabbits. Proc. Natl. Acad. Sci. U. S. A. 95:10134-10139.

63. Barron, M., Lake, R., Buda, A., and Tenaglia, A. 1997. Intimal hyperplasia after balloon injury is attenuated by blocking selectins. Circulation. 96:3587-3592.

64. Simon, D.I., et al. 2000. Decreased neointimal formation in Mac-1 -/mice reveals a role for inflammation in vascular repair after angioplasty. J. Clin. Invest. 105:293-300.

65. Kuman, A., Hoover, J., Simmons, C., Lindner, V., and Shebuski, R. 1997. Remodeling and neointimal formation in carotid artery of normal and P-selectin-deficient mice. Circulation. 96:4333-4342.

66. Kislinger, T., et al. 2001. RAGE mediates inflammation and enhanced expression of tissue factor in the vasculature of diabetic apolipoprotein E null mice. Arterioscler. Thromb. Vasc. Biol. 21:905-910

67. Friedman, J., et al. 1994. Advanced glycation endproducts activate the expression of monocyte and smooth cell chemoattractants by vascular smooth muscle cells. Circulation. 90:1567. (Abstr.)

68. Wu, H., Moulton, K., Horvai, A., Parik, S., and Glass, C. 1994. Combinatorial interaction between $\mathrm{AP}-1$ and ets domain proteins contribute to the developmental regulation of the MSR gene. Mol. Cell. Biol. 14:2129-2139. 